\title{
Comparative effects of argon green and krypton red laser photocoagulation for patients with diabetic exudative maculopathy
}

\author{
Moncef Khairallah, Raoudha Brahim, Mohamed Allagui, Nejib Chachia
}

\begin{abstract}
Aims/Background-Focal treatment of diabetic macular oedema is usually done using a haemoglobin absorbing wavelength, such as argon green laser. This study aimed to compare photocoagulation with argon green (514 $\mathrm{nm}$ ) and krypton red $(647 \mathrm{~nm})$, which is poorly absorbed by haemoglobin, in the focal treatment of patients with diabetic exudative maculopathy.

Methods-A total of 151 eyes of 78 outpatients were assigned randomly to receive either argon green $(n=79)$ or krypton red $(n=72)$ laser treatment. Pretreatment and post-treatment ocular examinations included visual acuity, fundus biomicroscopic examination, and fluorescein angiography. A total of 141 eyes of 73 patients were available for evaluation after 1 year follow up.

Results-No statistically significant difference was found between the two treatment groups with respect to visual acuity results, resorption of hard exudates, and resolution of focal retinal oedema.

Conclusion-Krypton red laser was effective in the treatment of diabetic exudative maculopathy. Our data suggest that differential absorption of the various wavelengths by haemoglobin within microaneurysms may not be an important factor for ultimate success of focal treatment.
\end{abstract}

(Br f Ophthalmol 1996; 80: 319-322)

Macular oedema is the most frequent cause of visual loss in patients with diabetic retinopathy. ${ }^{1}$ Numerous studies have shown the beneficial effect of photocoagulation in eyes with diabetic macular oedema. ${ }^{2-7}$ In the Early Treatment Diabetic Retinopathy Study (ETDRS), focal argon treatment to leaking microaneurysms and/or grid treatment to areas of diffuse leakage substantially reduced the risk of visual loss in patients with clinically significant macular oedema. ${ }^{89}$

Focal treatment of diabetic macular oedema is usually done using a haemoglobin absorbing wavelength, such as argon laser. ${ }^{18}$ The krypton red light $(647 \mathrm{~nm})$, which is poorly absorbed by haemoglobin, was not recommended for focal treatment. ${ }^{8}$

The aim of this prospective controlled study was to compare the efficacy of argon green and krypton red laser in the focal treatment of patients with diabetic exudative maculopathy.
Patients and methods

This clinical trial was undertaken between July 1992 and June 1994 and included 78 consecutive outpatients. Before entry into the study, all patients gave informed consent and the protocol was approved by the ethics committee of our institution. Inclusion criteria were a known type I or type II diabetes mellitus, and diabetic exudative maculopathy in one or both eyes with a best corrected visual acuity $\geqslant 20 / 100$. Diabetic exudative maculopathy was defined as the presence of hard exudates within 750 $\mu \mathrm{m}$ of the centre of the macula, associated with adjacent focal retinal oedema. Focal retinal oedema was defined as the presence of a focal area of retinal thickening caused by leaking microaneurysms. Patients with renal failure maintained on renal dialysis were excluded. Ocular exclusion criteria included all the following: proliferative diabetic retinopathy, previous laser photocoagulation, previous ocular surgery, significant media opacities, or any other ocular disease that was thought to interfere with assessment of treatment results.

The pretreatment ocular examination included a best corrected Snellen visual acuity measured and recorded by independent examiners, slit-lamp examination of the anterior segment, Goldmann applanation tonometry, examination of vitreous and retina with slit-lamp and contact lens, and fluorescein angiography of the retina. Grades of hard exudates were assessed according to the ETDRS classification. ${ }^{10}$

Eyes were randomised, using a sealed envelope system, to receive either argon green $(514 \mathrm{~nm})$ or krypton red $(647 \mathrm{~nm})$ laser treatment. The treatment was performed with topical anaesthesia in all cases. In all cases that required bilateral treatment, both eyes were treated during the same session.

Laser photocoagulation was directed to all leaky microaneurysms in areas of oedematous retina within 2 disc diameters of the centre of the macula. Treatment was restricted to microaneurysms further than $500 \mu \mathrm{m}$ from the centre of the macula. In focal argon green treatment, each lesion received a 50 to $100 \mu \mathrm{m}$ spot of $0 \cdot 10$ second duration, with adequate power to obtain definite whitening or darkening of the microaneurysms. Repeated applications to the same microaneurysm were usually needed. The focal krypton treatment was applied with 100 to $200 \mu \mathrm{m}$ spot of $0 \cdot 10$ second duration, with adequate power to obtain a moderate intensity burn. The treatment goal was to obtain whitening of the retina behind microaneurysms without whitening or 
Table 1 Baseline ophthalmic characteristics in treatment groups

\begin{tabular}{|c|c|c|c|}
\hline & $\begin{array}{l}\text { Argon } \\
(n=79 \text { eyes })\end{array}$ & $\begin{array}{l}\text { Krypton } \\
(n=72 \text { eyes })\end{array}$ & $\stackrel{p}{\text { Value }}$ \\
\hline \multicolumn{4}{|l|}{ Initial visual acuity } \\
\hline $20 / 20$ to $20 / 30$ & 37 & 34 & 0.96 \\
\hline $20 / 40$ to $20 / 60$ & 24 & 22 & 0.98 \\
\hline $20 / 70$ to $20 / 100$ & 18 & 16 & 0.93 \\
\hline \multicolumn{4}{|c|}{ Grade of hard exudates * } \\
\hline Grade 2 & 5 & 4 & 0.84 \\
\hline Grade 3 & 18 & 16 & 0.93 \\
\hline Grade 4 & 32 & 30 & 0.98 \\
\hline Grade 5 & 24 & 22 & 0.98 \\
\hline \multicolumn{4}{|l|}{ Presence of cystoid } \\
\hline macular oedema & 21 & 20 & $0 \cdot 86$ \\
\hline
\end{tabular}

darkening of the lesions themselves. Repeated burns were avoided.

Grid treatment to areas of diffuse macular oedema was not done during this study. Follow up examinations were performed every 4 months. These examinations were identical to the pretreatment examinations. Subsequent treatment of persisting focal retinal oedema was performed according to the same protocol. Evaluation of the treatment was based on its effects on visual acuity, hard exudates, and focal retinal oedema. A change in vision by at least two lines was required to be considered better or worse. Visual acuity that remained the same or varied by one line was considered stable. Effects of treatment on hard exudates were classified in three groups: complete resorption, partial resorption, and same or increase. Complete or partial resolution of focal retinal oedema was considered if thickening of treated areas had completely or partially disappeared. The number of laser sessions required and complications related to the treatment were also recorded for each eye.

All variables in this study were expressed by their means and ranges. Comparison between the argon group and the krypton group was made using the $\chi^{2}$ test with a probability level of 0.05 used to declare statistical significance.

\section{Results}

This study was conducted in 151 eyes of 78 patients ( 35 men and 43 women). Ages ranged from 34 to 72 years (average 55.7), and duration of diabetes from 3 to 27 years (average 12 years). Insulin was used by 32 patients, and oral hypoglycaemic agents by 46 patients. Twenty one patients had a history of systemic hypertension.

Argon green laser treatment was applied to 79 eyes and krypton red laser treatment to 72 eyes. Seventy four patients were treated bilaterally: in 70 patients, one eye was treated with argon green and the other eye with krypton red; three patients had both eyes treated

Table 2 Visual results for number of eyes(\%)*

\begin{tabular}{llll}
\hline & $\begin{array}{l}\text { Argon }(n=73) \\
\text { No (\%) }\end{array}$ & $\begin{array}{l}\text { Krypton }(n=68) \\
\text { No (\%) }\end{array}$ & $\begin{array}{l}p \\
\text { Value }\end{array}$ \\
\hline Improved & $13(18)$ & $13(19)$ & 0.84 \\
Stable & $55(75)$ & $51(75)$ & 0.96 \\
Worse & $5(7)$ & $4(6)$ & 0.81 \\
\hline
\end{tabular}

^At 1 year follow up evaluation.
Table 3 Effect of treatment on hard exudates for number of eyes (\%)*

\begin{tabular}{llll}
\hline & $\begin{array}{l}\text { Argon }(n=73) \\
\text { No (\%) }\end{array}$ & $\begin{array}{l}\text { Krypton }(n=68) \\
\text { No (\%) }\end{array}$ & $\begin{array}{l}p \\
\text { Value }\end{array}$ \\
\hline Total resorption & $54(74)$ & $49(72)$ & $0 \cdot 79$ \\
Partial resorption & $18(25)$ & $18(27)$ & 0.80 \\
Same or increase & $1(1)$ & $1(1)$ & 0.95 \\
\hline
\end{tabular}

^At 1 year follow up evaluation.

with argon green, and one patient had both eyes treated with krypton red. Both treatment groups were similar with respect to all of the following: initial visual acuity, grades of hard exudates, and the presence of associated cystoid macular oedema (Table 1). A total of 141 eyes of 73 patients were available for evaluation after 1 year follow up. Two patients died and three patients were lost to follow up. These five patients were treated bilaterally: in four patients, one eye was treated with argon green and the other eye with krypton red; one patient had both eyes treated with argon green.

Visual results at the 1 year follow up evaluation are reported in Table 2 . Visual acuity remained stable or improved in 68 of 73 eyes $(93 \%)$ treated with argon green, and in 64 of 68 eyes (94\%) treated with krypton red. Worsening of visual acuity was shown in five of 73 eyes $(7 \%)$ treated with argon green, and in four of 73 eyes $(6 \%)$ treated with krypton red. No statistically significant difference in visual outcome was found between the argon green and the krypton red groups.

Table 3 compares the outcome of hard exudates for all treated eyes. Fifty four of 73 eyes $(74 \%)$ treated with argon green had total resorption of hard exudates, compared with 49 of 68 eyes $(72 \%)$ treated with krypton red. Partial resorption of hard exudates occurred in 18 of 73 eyes $(25 \%)$ in the argon green treated group, and in 18 of 68 eyes $(27 \%)$ in the krypton red treated group. The difference in the efficacy on the resorption of hard exudates between the two types of laser was not statistically significant.

Table 4 lists a comparison of treatment effects on focal retinal oedema. Fifty nine of 73 eyes $(81 \%)$ treated with argon green had complete resolution of focal retinal oedema, compared with 53 of 68 eyes $(78 \%)$ treated with krypton red. Thirteen of 73 eyes $(18 \%)$ treated with argon green and 14 of 68 eyes $(21 \%)$ treated with krypton red had partial resolution of focal retinal oedema. The difference in the efficacy on the resolution of focal retinal oedema between the two types of laser was not statistically significant.

Fifty six of 73 eyes $(77 \%)$ treated with argon green, and 51 of 68 eyes $(75 \%)$ treated with

Table 4 Effect of treatment on focal oedema for number of eyes $(\%)^{\star}$

\begin{tabular}{llll}
\hline & $\begin{array}{l}\text { Argon }(n=73) \\
\text { No (\%) }\end{array}$ & $\begin{array}{l}\text { Krypton }(n=68) \\
\text { No (\%) }\end{array}$ & $\begin{array}{l}p \\
\text { Value }\end{array}$ \\
\hline Complete resolution & $59(81)$ & $53(78)$ & 0.67 \\
Partial resolution & $13(18)$ & $14(21)$ & 0.67 \\
Same or increase & $1(1)$ & $1(1)$ & 0.95 \\
\hline
\end{tabular}

^At 1 year follow up evaluation. 
krypton red had a single laser treatment session. Seventeen of 73 eyes (23\%) in the argon green group had two treatment sessions, compared with 17 of 68 eyes (25\%) in the krypton red group.

No complication was recorded during the study, except in one patient submacular fibrosis developed after focal argon green treatment.

\section{Discussion}

The current study shows that the krypton red laser, a poorly haemoglobin absorbed wavelength, was as effective as the argon green laser, which is well absorbed by haemoglobin, in the focal treatment of patients with diabetic exudative maculopathy. No statistically significant difference was found between the two wavelengths with respect to visual outcome, resorption of hard exudates, and resolution of focal retinal oedema.

Focal argon green laser treatment was performed according to the protocol recommended by the ETDRS for focal leaks. ${ }^{89}$ The desired endpoint of such treatment is a whitening or darkening of leaking microaneurysms to achieve closure or obliteration of these lesions. Focal krypton red laser treatment was applied in our study just to produce a moderate intensity burn, deep to each leaking microaneurysm.

According to the current concept of focal treatment in diabetic macular oedema, the efficacy of krypton red light was unexpected. It is widely assumed that focal treatment must produce ablation of leaking retinal microaneurysms to obtain resolution of diabetic macular oedema. ${ }^{19}$ The generally believed mechanism by which focal treatment works is absorption of laser light by haemoglobin within microaneurysms. Therefore, the use of a haemoglobin absorbing wavelength such as argon laser was considered necessary. Argon blue-green laser was widely used and its benefit was demonstrated in many major clinical trials of photocoagulation for diabetic macular oedema. 23 5-8 Nevertheless, the blue wavelength has several undesirable effects for macular photocoagulation, such as retinal toxicity, inefficient scatter, risk of cataractogenesis, and potential for inner retinal damage from absorption by macular xanthophyll. ${ }^{11}$ For these reasons, the argon green laser wavelength has supplanted the blue-green laser in common clinical usage.

Two recent uncontrolled studies reported the efficacy of non-haemoglobin absorbing wavelengths in focal treatment of clinically significant diabetic macular oedema. ${ }^{1213}$ In the first study, ${ }^{12}$ focal treatment was performed with krypton red laser. In the second study, ${ }^{13}$ focal treatment was done using a diode laser near infrared radiation $(810 \mathrm{~nm})$. Data from our controlled clinical trial demonstrated that focal treatment can be successfully achieved using a non-haemoglobin absorbing wavelength, without coagulation of the microaneurysms. Even in focal treatment with a haemoglobin absorbing wavelength, such as argon green, direct coagulation of a microaneurysm could hardly be achieved, because the haemoglobin level is low in the lesion. ${ }^{14}$ It is therefore unlikely that the beneficial effect of focal treatment is mainly related to haemoglobin absorption of laser light within the microaneurysm. A clinicopathological correlation of focal argon treatment in a diabetic patient treated as part of the ETDRS has been reported. ${ }^{15}$ It was interesting to note that the majority of retinal burns involved the retinal pigment epithelium, and photoreceptor layer, but spared the inner nuclear layer, within which microaneurysms locate. However, successful closure of many of the microvascular lesions was observed. Resorption of hard exudates and resolution of focal retinal oedema could be attributed to other beneficial tissue responses secondary to laser photocoagulation. These include regeneration of the retinal pigment epithelium resulting in restoration of the outer blood-retinal barrier ${ }^{16}$; stimulation of the vascular repair process resulting in repair of the inner blood-retinal barrier ${ }^{17}$; improvement of retinal oxygenation leading to autoregulatory vasoconstriction $^{18}$; and healing of the outer plexiform layer where oedema accumulates. ${ }^{14}$

The current study tends to confirm the concept that differential absorption of various wavelengths by haemoglobin within retinal microaneurysms has little to do with the ultimate success of focal treatment of diabetic macular oedema. We agree with Ulbig et al ${ }^{13}$ that the current protocol in relation to focal macular photocoagulation demands power levels that are likely to be in excess of those necessary to produce a beneficial effect. Using repeated small size short duration and intense burns to achieve ablation of microaneurysms is probably unnecessary, and should be avoided to minimise the risk of iatrogenic complications, such as choroidal neovascularisation, submacular fibrosis, and choriovitreal proliferation. ${ }^{19-21}$

1 Bresnick GH. Diabetic macular edema. Ophthalmology 1986; 93: 989-97.

2 Patz A, Schatz H, Berkow JW, Gittelsohn AM, Ticho U. Macular edema: an overlooked complication of diabetic retinopathy. Trans Am Acad Ophthalmol Otolaryngol 1973; 77: $34-42$.

3 Blankenship GW. Diabetic macular edema and argon laser photocoagulation: a prospective randomized study. Ophthalmology 1979; 86: 69-78.

4 Townsend C, Bailey J, Kohner E. Xenon arc photocoagulation for the treatment of diabetic maculopathy. Interim report of multicenter controlled clinical study. $\mathrm{Br} \mathcal{J}$ Ophthalmol 1980; 64: 385-91.

5 Reeser F, Fleischman J, Williams GA, Goldman A. Efficacy of argon laser photocoagulation in the treatment of circinate diabetic retinopathy. Am $\mathcal{f}$ Ophthalmol 1981; 92: 762-7.

6 McDonald RH, Schatz H. Grid photocoagulation for diffuse macular edema. Retina 1985; 5: 65-72.

7 Olk J. Modified grid argon (blue-green) laser photocoagulation for diffuse diabetic macular edema. Ophthalmology 1986; 93: 938-50.

8 Early Treatment Diabetic Retinopathy Study Research Group. Photocoagulation for diabetic macular edema: Early Treatment Diabetic Retinopathy Study report number 1. Arch Ophthalmol 1985; 103: 1796-806.

9 Early Treatment Diabetic Retinopathy Study Research Group. Treatment techniques and clinical guidelines for photocoagulation of macular edema: report number 2 . Ophthalmology 1987; 94: 761-74.

10 Early Treatment Diabetic Retinopathy Study Research Group. Grading diabetic retinopathy from stereoscopic color fundus photographs. An extension of the modified Airlie House classification ETDRS Report Number 10. Ophthalmology 1991; 98: 786-806.

11 Mainster MA. Wavelength selection in macular photocoagulation. Tissue optics, thermal effects, and laser systems. Ophthalmology 1986; 93: 952-8.

12 Chew EY. Treatment of diabetic macular edema with krypton laser photocoagulation. In: Gitter KA, Schatz H ton laser photocoagulation. In: Gitter KA, Schatz H,
Yannuzzi LA, McDonald HR, eds. Laser photocoagulation 
of retinal disease. From the International Laser Symposium of the Macula. San Francisco: Pacific Medical Press, 1988: 83-7.

13 Ulbig MW, McHugh AMc, Hamilton AMP. Diode laser photocoagulation for diabetic macular oedema. $\mathrm{Br} \mathcal{F}$ Ophthalmol 1995; 79: 318-21.

14 Haut J, Limon S, Massin M, Perdriel G. Le laser en ophtalmologie. Rapport Soc Fr Ophtalmol. Paris: Masson, 1981.

15 Wallow IHL, Bindley CD. Focal photocoagulation of diabetic macular edema. A clinicopathologic case report. Retina 1988; 8: 261-9.

16 Wallow IHL. Repair of the pigment epithelial barrier following photocoagulation. Arch Ophthalmol 1984; 102: 126-35.

17 Marshall J, Clover G, Rothery S. Some new findings on retinal irradiation by krypton and argon lasers. Doc Ophthalmol Proc Ser 1984; 36: 21-37.

18 Gottfredsdottir MS, Stefansson E, Jonasson F, Gislason I. Retinal vasoconstriction after laser treatment for diabetic macular edema. Am $f$ Ophthalmol 1993; 115: diabetic macular edema. Am 7 Ophthalmol 1993; 115:

19 Lewis H, Schachat AP, Haimann MH, Haller JA, Quinlan $\mathrm{P}$, von Fricken MA, et al. Choroidal neovascularisation after laser photocoagulation for diabetic macular edema. Ophthalmology 1990; 97: 503-11.

20 Han DP, Mieler WF, Burton TC. Submacular fibrosis after photocoagulation for diabetic macular edema. $A m \mathcal{F}$ Ophthalmol 1992; 113: 513-21.

21 Benson WE, Townsend RE, Pheasant TR. Choriovitreal and subretinal proliferations. Complications of photocoagulation. Ophthalmology 1979; 86: 283. 